\title{
CUA guidelines on prostate biopsy methodology
}

\author{
Assaad El-Hakim, MD, FRCSC,; Sabri Moussa, MD ${ }^{\dagger}$
}

\section{Introduction}

Transrectal ultrasound prostate biopsy (TRUS Bx) is increasingly performed by urologists. Lee and colleagues reported on the diversity in TRUS Bx practice and training in United Kingdom. ${ }^{1}$ Fifty-six percent of the surveyed urologists were actively involved in TRUS Bx and $68 \%$ of them did not think they received enough training. There is a wide variation in patient preparation (antibiotic prophylaxis regimens and analgesia used), biopsy schemes and indications for repeat biopsy. The lack of standardized guidelines for TRUS Bx highlights the necessity of a structured program for training the new generation of urologists.

\section{A. Patient preparation}

Patients should be informed of the risks and benefits of the TRUS Bx and informed written consent should be obtained.

\section{Antiplatelets and anticoagulants}

Most practitioners recommend discontinuation of antiplatelet agents (acetylsalicylic acid [ASA] and products containing ASA, such as mesalamine, clopidogrel, ticlodipine, and nonsteroidal anti-inflammatory drugs [NSAIDs]) before TRUS Bx to minimize the risk of bleeding complications. It is recommended to stop the use of ASA/NSAIDs 3 to 5 days before the biopsy. Clopidogrel needs to be stopped 7 days and ticlodipine needs to be stopped 14 days before TRUS Bx. This practice is based on the experience of interventions at other sites, which may or may not be applicable to prostate sampling. Of note, prospective studies on TRUS Bx with continued use of low-dose ASA revealed that there was no increased risk of overall bleeding or hematuria ${ }^{2-4}$ (Level 2 evidence).

The lack of evidence on post-TRUS Bx hemorrhagic complications in patients taking warfarin, and the perceived high risk of occurrence of such complications would suggest a conservative stance with regard to the discontinua-

tion of warfarin prior to biopsy. It is suggested to discontinue warfarin except in those patients at high risk of thromboembolic events at which time bridging therapy with heparin is suggested. A survey among urologists and radiologists found that $84 \%$ of urologists stopped warfarin 4 days before TRUS Bx and $95 \%$ of radiologists stopped it 5 days before TRUS Bx. ${ }^{5}$ An international normalized ratio below 1.5 is accepted for most elective procedures. ${ }^{6}$ The decision whether to stop anticoagulants depends on the indications for anticoagulation and the risks of thrombosis in a particular patient. This decision should be discussed with the patient and the primary physician managing the anticoagulant.

A number of recommendations were offered for the perioperative management of patients on warfarin therapy according to the risk of thrombosis and indications for anticoagulation. ${ }^{6}$ Patients who had acute venous or arterial thromboembolism during the month before the procedure may have the inpatient procedure and be switched to bridging therapy with intravenous (IV) heparin before and after the procedure. Those patients with other indications (mechanical heart valve, recurrent venous thromboembolism or nonvalvular atrial fibrillation) and lower risk, can be switched to subcutaneous heparin or low molecular weight heparin. ${ }^{6}$ The relation between warfarin use and the frequency of bleeding complications after the TRUS Bx was reported in a prospective study of 1000 patients. Forty-nine patients continuously used warfarin before and after the biopsy. The prevalence and severity of bleeding complications were assessed by a questionnaire 10 days after the biopsy. There was no significant difference in the severity of bleeding between patients taking warfarin and controls ${ }^{7}$ (Level 2 evidence). Limitations of the aforementioned study include non-randomized design, patients had either 6- or 4-core biopsies, life-threatening hemorrhagic complications may have been missed due to small sample size, recall bias must be considered as complications were entered retrospectively 10 days after biopsy, and patients on warfarin may underestimate severity of hemorrhagic complications. In order to change the practice of stopping anticoagulants before the TRUS Bx, further studies are needed. Since these studies are currently unavailable, best practice would entail a safe conservative approach detailed above. 
Recommendations: The indication for the antiplatelet agent has to be reviewed with the patient, his primary care physician or cardiologist and only after that should the antiplatelet agent be stopped. Antiplatelets (i.e., ASA, clopidogrel and ticlodipine) should be stopped 7 to 14 days prior to biopsy (Grade B recommendation). Anticoagulants (i.e., warfarin) should be stopped 4 to 5 days prior. Bridging therapy with IV heparin or low molecular weight heparin should be considered in high-risk patients (Grade of recommendation B).

\section{Cleansing enema}

Patients may be advised to self-administer a cleansing enema at home before the biopsy.

Enema use was reported by about $80 \%$ of urologists surveyed regarding patient preparation for TRUS Bx. 8,9 This may produce a superior acoustic window for prostate imaging as a result of decreasing the amount of feces in the rectum, and may be more comfortable for some individuals. The effect on infection reduction is debatable. Many large centres have abandoned the use of cleansing enemas citing lack of data supporting its usage, patient cost and inconvenience. To address the role of an enema in preventing infection, Lindert and colleagues analyzed many variables, including bacteriuria, bacteraemia and organisms cultured from the biopsy needle in a randomized study of 50 men (25 received pre-biopsy enema and 25 no enema). ${ }^{10}$

Bacteremia was reported in $4 \%$ of patients given an enema compared to $28 \%$ of patients who had no enema. However, bacteremia was asymptomatic in both groups. Biopsy needle cultures had the same incidence of positive findings. The authors concluded that asymptomatic bacteraemia may be significantly minimized by a pre-biopsy enema independent of antibiotic administration ${ }^{10}$ (Level 1 evidence). The clinical significance of these findings is yet to be defined.

Recommendation: There is no strong evidence to recommend for or against the use of enema (Grade $A$ recommendation).

\section{Antibiotic prophylaxis}

Different regimens using oral and IV antibiotics have been studied..$^{11-17}$ The post-biopsy duration of oral antibiotics is controversial. Several studies examined the use of one dose of an oral fluoroquinolone 30 to 60 minutes before biopsy with continued therapy for 2 to 3 days $^{13,15}$ (Level 2 evidence) versus single-dose oral fluoroquinolones (Level 1 evidence). ${ }^{14,17}$ Both regimens resulted in minimal infectious complications. Another accepted regimen is IV ampicillin (vancomycin in cases of penicillin sensitivity) and gentamicin before the procedure followed by oral flouroquinolones for 2 to 3 days. The latter regimen is suggested for patients at risk of developing endocarditis or infection of cardiac prosthetics, such as pacemakers and implanted cardiac defibrillators ${ }^{16}$ (Level 4 evidence). It has also been shown that antibiotic prophylaxis lowers the risk of infection with multiple core biopsies. The widespread use of flouroquinolones to treat urinary tract infections increased the rate of fluoroquinolone-resistant Escherichia coli. It was reported that the causative pathogen in urinary tract infection after TRUS Bx was mainly Escherichia coli with high resistance rates to fluoroquinolones. ${ }^{18}$ Adding IV aminoglycoside to fluoroquinolones prophylactic regimens may minimize the incidence of urinary tract infection after TRUS $B x^{18,19}$ (Level 3 evidence) in institutions where this problem has been documented.

Recommendation: Broad-based gram-negative antibiotic prophylaxis (e.g., fluorquinolone) should be administered prior to biopsy and may be continued for 2 to 3 days postbiopsy (Grade B recommendation). However, many centres have moved towards shorter courses of antibacterial prophylaxis especially with the availability of single-dose long-acting fluoroquinolones citing patient cost, inconvenience and the paucity of data demonstrating superiority with multiday dosing regimens.

\section{Analgesia}

Although TRUS Bx is well-tolerated, it is associated with pain when performed without anesthesia ${ }^{20}$ (Level 3 evidence), especially with the increased number of cores performed. The most commonly used anaesthetic is lidocaine either in gel suspension or an injectable preparation (periprostatic nerve block). Periprostatic nerve block (PPNB) requires $1 \%$ or $2 \%$ lidocaine without epinephrine, and a long spinal needle (7-inch, 22-gauge). Various methodologies for injection sites and quantities have been described, and the most quoted and used protocol uses $5 \mathrm{~mL}$ of the lidocaine injected bilaterally in region of the prostatic vascular pedicle at the base of the prostate just lateral to the junction between the prostate and seminal vesicle. ${ }^{21}$ Intrarectal lidocaine gel failed to show improvement in pain control over placebo ${ }^{22}$ (Level 1 evidence). However, several studies documented that periprostatic infiltration with lidocaine around the nerve bundles provides satisfactory pain control ${ }^{23-25}$ (Level 1 evidence). Pain scores are significantly decreased from an average of 3.7 to 5.5 in controls compared to 0.5 to 2.4 for PPNB. The morbidity associated with PPNB was first assessed in a prospective study that reported no significant difference in the incidence of urethral bleeding, rectal bleeding or fever in the PPNB group compared to the control group. However, asymptomatic bacteriuria was significantly reported in the PPNB group ${ }^{26}$ (Level 1 evidence). In an attempt to circumvent PPNB, different methods of analgesia were 
reported, namely oral narcotic analgesia and intramuscular NSAIDs ${ }^{27,28}$ (Level 1 evidence).

Pain control with oral and intramuscular analgesics was not statistically different from control groups, therefore these methods were abandoned. The analgesic effect of intrarectal diclofenac suppository was also assessed in randomized control trials. Diclofenac suppositories (100 mg) significantly reduced pain scores compared with placebo, but to a lesser degree than PPNB did.

The average pain score with diclofenac suppository was 2.8 to 3.4 compared to 4.9 to 5.9 for placebo ${ }^{29,30}$ (Level 1 evidence). Therefore, PPNB provides better analgesia than NSAID suppositories and should be considered as a first choice $^{23-25}$ (Level 1 evidence).

\section{Recommendation: Periprostatic nerve block is highly rec- ommended especially with an extended core biopsy scheme (Grade $A$ recommendation).}

\section{Patient positioning}

Patients are usually placed in the left lateral decubitus position with knees and hips flexed at 90 degrees. The buttocks should be flush with the edge of the table to allow manipulation of the probe and biopsy gun without obstruction. Depending on surgeon handedness and preference the right lateral decubitus or lithotomy position can be used (Level 4 evidence).

\section{B. Labelling and processing}

There is controversy around the processing and submission of TRUS Bx specimens. One option is placing multiple ipsilateral biopsies in a single container (left- and rightsided specimens). ${ }^{16,31}$ This often entangles the biopsies and may result in $40 \%$ of the tissue surface area being lost, with only a 5-degree shift in angle of the needle biopsy within the tissue block. ${ }^{32}$ This increases equivocal reports, which then require repeat biopsy. A second option is using multipack container kits ${ }^{31,33}$ which are technically more complex and costly ${ }^{34}$ but, in at least one study, decreases the equivocal diagnosis rate (atypical glands and ASAP) ${ }^{35}$ (Level 3 evidence). Many leading genitourinary pathologists recommend multipack containers to reduce errors and subsequent risk of repeat biopsy. With the advancement of image-guided therapies and future focal therapies (brachytherapy, cryotherapy, high-intensity focused ultrasound) as well as nerve-sparing radical surgery, the location of cancer at biopsy has become important and assumes a prominent role in pretreatment planning.

\section{Prostate biopsy schemes}

Prostate examination with an evaluation of prostate volume, imaging of both transverse and sagittal planes prior to the biopsy is necessary. The examination usually starts at the base of the gland and extends to the apex, noting the location and characteristics of any lesion (i.e., hypoechoic and hyperechoic lesions, calcifications, contour abnormalities and cystic structures).

Seminal vesicles (SV) are also examined for evidence of invasion with loss of SV angle, SV dilatation and echogenicity.

Material for histopathological examination obtained by ultrasound-guided transrectal 18-gauge core biopsy has become the standard. A spring biopsy device or biopsy gun passed through the needle guide attached to the ultrasound probe is most often used. Biopsy needle path has the best visualization in the sagittal plane; with the advent of biplanar ultrasound technologies, simultaneous transverse and sagittal imaging is possible and can be helpful in needle localization and placement. The biopsy gun advances the needle $0.5 \mathrm{~cm}$ and samples the subsequent $1.5 \mathrm{~cm}$ or $2 \mathrm{~cm}$ of tissue with the tip extending $0.5 \mathrm{~cm}$ beyond the area sampled..$^{36}$

Biopsies are obtained as lesion-guided or systematic cores. Lesion-guided biopsies can be used for palpable nodules or ultrasound detected lesions. In one study, lesion-guided biopsies using contrast enhanced colour Doppler detected cancers as much as 10 times that of systematic biopsies alone, ${ }^{37}$ but the method has not yet gained widespread acceptance or availability. The limitations in cancer detection with lesion-guided biopsy has led to the emergence of systematic TRUS Bx techniques. Since this technique was first described in 1989, ${ }^{38}$ there has been no consensus on the ideal number of cores and location for the best cancer yield. The standard sextant scheme gave rise to a broad variety of biopsy methods that can be generally grouped under the widely accepted 5-region anatomical model. The latter defines 2 paramedian regions (traditional sextant), 2 lateral regions and 1 central region.

\section{Sextant biopsy scheme}

The original systematic biopsy method is the sextant biopsy scheme ( 1 core from the base, mid, and apex bilaterally). ${ }^{38}$ With this scheme, the cores were taken through the parasagittal plane, which resulted in some false-negative results ${ }^{39}$ (Level 2 evidence). Up to $30 \%$ of cancers were missed by the standard sextant biopsy ${ }^{40,41}$ (Level 2 evidence).

\section{Extended biopsy schemes}

To improve the cancer detection rate, Stamey and colleagues suggested laterally directed biopsies as $75 \%$ of prostate cancers originate from the peripheral zone. ${ }^{42}$ Five-region prostate biopsy in which additional cores are obtained from the far lateral peripheral zone and midline in addition to 
the standard sextant biopsy was described in 1997. ${ }^{39}$ Several groups have published results showing higher cancer detection rates with the 5-region prostate biopsy scheme compared to standard sextant technique for primary biopsy (cores ranged from 10 to 13$)$. $^{39,43-47}$

An exhaustive systematic review of the literature of cancer detection rates with different extended prostate biopsy schemes compared to the standard sextant scheme was published by Eichler and colleagues $^{48}$ (Level 1 evidence). Eighty-seven studies were reviewed with a total of 20698 patients. The number of cores reported in individual studies ranged from 6 to 22 cores. Schemes with 12 cores showed a relative positivity rate of 1.31 compared to standard sextant scheme. The highest relative positivity rate (1.48) was reported with the 18 to 22 schemes of the 5-region biopsy pattern. However, multivariate analysis revealed no significant difference between 18 to 22 core schemes, 12-core schemes or 10 -core schemes in cancer detection. ${ }^{48}$ Adverse events reported with extended core schemes (10 to 12 cores) were not statistically diferent from that of sextant schemes. However, schemes with more than 12 cores resulted in significant increases in TRUS Bx adverse events. Extended prostate biopsy schemes consisting of 12 cores, including standard sextant biopsy scheme and laterally directed cores strike the balance between cancer detection and adverse events $^{48}$ (Level 1 evidence).

Pepe and Argona evaluated prostate cancer detection rate in patients who underwent saturation prostate biopsy (24 to 37 cores) as primary biopsy. ${ }^{49}$ Cancer detection rate was not statistically different with saturation biopsy $(46.9 \%)$ compared to 12 -core biopsy $(39.8 \% ; p=0.3)$ and the 18 core biopsy $(49 \% ; p=0.6)^{49}$ (Level 3 evidence). Saturation prostate biopsy is not recommended as a primary biopsy scheme, as it did not significantly increase prostate cancer detection rates compared to 12-core biopsy schemes. Toi and colleagues suggested adding targeted biopsy in the presence of prostate lesions to the systematic biopsy schemes to improve cancer detection rates. The presence of a lesion increased the likelihood of cancer detection $(57.8 \% \mathrm{vs}$. $30.8 \%$ ). Biopsies from these lesions have a greater volume of cancer detected in each positive core and a higher grade of cancer. ${ }^{50}$

Recommendation: An extended biopsy scheme of 10 to 12 cores is recommended to optimize the ratio of cancer detection to adverse post-biopsy events. Lesion-guided biopsy can be added to further optimize cancer detection (Grade $A$ recommendation).

\section{Impact of prostatic volume on prostate biopsy technique}

Calculating prostate gland volume is a routine part of every TRUS Bx session and an indirect relationship has been demonstrated between prostate volume and the likelihood of detecting prostate cancer. ${ }^{51}$ Prostate cancer detection with standard sextant scheme in glands larger than $50 \mathrm{cc}$ was $23 \%$ compared to $38 \%$ in smaller glands ${ }^{52}$ (Level 3 evidence). Different studies reported that the cancer detection rates are related conversely to the prostate gland volume: the larger the gland, the lower cancer detection rates regardless of the biopsy scheme used ${ }^{53-5}$ (Level 3 evidence). Several mathematical models (nomograms and tables) were developed to determine the minimum number of cores necessary to preclude missing significant cancers in various size glands over a wide range of serum prostate-specific antigen (PSA) and patient age. ${ }^{56}$ Generally, a minimum of 10 cores was found to be necessary for prostate volumes $30 \mathrm{cc}$ and above.

\section{Recommendations: Mathematical formulas that account for prostate size, patient age and PSA range are not required provided an extended biopsy scheme is applied (Grade B recommendation).}

\section{Transition-zone biopsies}

Transition zone is the site of origin for about $15 \%$ of prostate adenocarcinomas; however, isolated transition-zone tumours detected on prostate biopsy are uncommon. Cancer detection rates increases by $1.8 \%$ to $4.3 \%$ upon adding transitionzone biopsies to the primary biopsy, but there is little evidence to recommend routine transition-zone sampling $57-60$ (Level 2 evidence). Transition-zone biopsies may be indicated in two situations: (1) in men with gland size of more than $50 \mathrm{~mL}\left(15 \%\right.$ increases in cancer yield) ${ }^{47}$ (Level 2 evidence) and (2) in patients in whom systematic biopsies failed to reveal cancer with markedly elevated or rapidly increasing PSA ${ }^{58}$ (Level 2 evidence).

\section{Recommendations: Transition-zone biopsies are seldom necessary and add little to the overall detection rate of an extended biopsy scheme (Grade $B$ recommendation).}

\section{Repeated biopsies}

Negative prostatic biopsy with rising PSA levels or the presence of suspicious prostatic lesions, high-grade prostatic intraepithelial neoplasia (HGPIN) or atypical small acinar proliferation (ASAP) are challenging dilemmas facing urologists. Cancer detection rates in repeat biopsy populations depend on the number and location of cores obtained. In one study, cancer detection rates were $39 \%$ and $28 \%$ in patients who underwent prior standard sextant and extended biopsy schemes, respectively ${ }^{61}$ (Level 3 evidence). 


\section{a) High-grade prostatic intraepithelial neoplasia}

High-grade prostatic intraepithelial neoplasia is thought to be a precursor to invasive Adenocarcinoma. ${ }^{62}$ During the sextant biopsy scheme era, the cancer detection rate on repeat biopsy for HGPIN was 25\% to 70\%. ${ }^{63-67}$ High-grade prostatic intraepithelial neoplasia may be considered a component of a limited field effect, and its presence suggests that cancer might exist elsewhere in the gland. Sampling of the prostate with extended biopsy schemes is more likely to find that cancer. With the introduction of extended biopsies the cancer detection rates on first repeat biopsy for HGPIN decreased dramatically to $2.3 \%,{ }^{68} 4 \%,{ }^{69} 4.5 \%{ }^{70}$ (Level 3 evidence) in three contemporary series; these rates are no higher than the rate of cancer detection on repeat biopsy of normal findings on first biopsy. In the current era of extended biopsy schemes, HGPIN is no longer considered a strict indication for repeat biopsy and patients should be followed clinically with PSA and digital rectal examination (DRE).

\section{b) Atypical small acinar proliferation}

Atypical small acinar proliferation (ASAP) findings should be viewed differently than HGPIN. Atypical small acinar proliferation is a focus of morphologic malignant cells with equivocal basal cell layer. ${ }^{71}$ It may result from insufficient material or tissue processing and the pathologist is uncomfortable labelling it invasive cancer. Cancer detection rates on repeat biopsy for ASAP found on sextant biopsies was $40 \%$ to $50 \%{ }^{62}$ Using an extended core biopsy scheme, the cancer detection rate remained as high as $36 \%{ }^{70}$ to $59.1 \%^{72}$ on first repeat biopsy and $16 \%$ on second repeat biopsy. ${ }^{70}$ Because most cancers were found in the same region as the ASAP on repeat biopsy, and because $20 \%$ to $45 \%$ of cancers can be found outside the area of ASAP, $66,72,73$ a systematic re-biopsy of the prostate is recommended with additional targeted cores (Level 3 evidence).

Different prostate biopsy techniques were used to minimize false negative biopsies in repeat biopsy populations.

- Saturation biopsy is an aggressive biopsy scheme with as many as 45 cores obtained. ${ }^{74}$ The incidence of prostate cancer at the second biopsy using saturation biopsy scheme versus 18 -core set was $22.6 \%$ versus $10.9 \%(p=0.02)$. At the third biopsy, the incidence of prostate cancer with saturation biopsy scheme versus 18 -core set was $6.2 \%$ versus $0 \%(p=0.01)^{49}$ (Level 3 evidence). This technique requires regional or general anaesthesia and may require hospital admission. ${ }^{75}$

Saturation biopsy may be considered in high-risk cases (e.g., rising PSA, abnormal DRE, persistent ASAP) with at least 2 previous negative extended biopsies (Grade $B$ recommendation).
- Transperineal template technique is another aggressive scheme for repeat biopsy. In one study, a mean of 15.1 biopsy samples were obtained with a cancer detection rate of $43 \%$ in a high-risk group of patients ${ }^{76}$ (Level 3 evidence).

Recommendations: Atypical small acinar proliferation lesions are cancerous until proven otherwise and should undergo repeat biopsy (Grade B recommendation). Repeat biopsy may no longer be indicated for HGPIN lesions in the era of extended core biopsy, unless the patient has an increase in PSA or change on DRE (Grade B recommendation).

*Assistant Professor of Urology, McGill University, Montréal, QC; ${ }^{\dagger}$ McGill University Health CentreResearch Institute, Montréal, QC

Competing interests: None declared.

This paper has been peer-reviewed.

\section{References}

1. Lee G, Attar K, Laniado M, et al. Trans-rectal ultrasound guided biopsy of the prostate: nationwide diversity in practice and training in the United Kingdom. Int Urol Nephrol 2007;39:185-8.

2. Maan Z, Cutting $\mathrm{CW}$, Patel $\mathrm{U}$, et al. Morbidity of transrectal ultrasonography-guided prostate biopsies in patients after the continued use of low-dose aspirin. BJU Int 2003;91:798-800.

3. Rodriguez LV, Terris MK. Risks and complications of transrectal ultrasound-guided prostate needle biopsy: a prospective study and review of the literature. J Urol 1998;160:2115-20.

4. Herget EJ, Saliken JC, Donnelly BJ, et al. Transrectal ultrasound-guided biopsy of the prostate: relation between ASA use and bleeding complications. Can Assoc Radiol J 1999;50:173-6.

5. Connor SE, Wingate JP. Management of patients treated with aspirin or warfarin and evaluation of haemostasis prior to prostatic biopsy: a survey of current practice amongst radiologists and urologists. Clin Radiol 1999;54:598-603.

6. Kearon C, Hirsh J. Management of anticoagulation before and after elective surgery. N Engl J Med 1997;336:1506-11.

7. Ihezue CU, Smart J, Dewbury KC, et al. Biopsy of the prostate guided by transrectal ultrasound: relation between warfarin use and incidence of bleeding complications. Clin Radiol 2005;60:459-63.

8. Shandera KC, Thibault GP, Deshon GE Jr. Variability in patient preparation for prostate biopsy among American urologists. Urology 1998;52:644-6.

9. Davis $M$, Sofer M, Kim SS, et al. The procedure of transrectal ultrasound guided biopsy of the prostate: a survey of patient preparation and biopsy technique. J Urol 2002;167:566-70.

10. Lindert KA, Kabalin IN, Terris MK. Bacteremia and bacteriuria after transrectal ultrasound guided prostate biopsy. J Urol 2000;164:76-80.

11. Aus $G$, Ahlgren $G$, Bergdahl $S$, et al. Infection after transrectal core biopsies of the prostate- -risk factors and antibiotic prophylaxis. Br J Urol 1996;77:851-5.

12. Collins $\mathrm{GN}$, Lloyd $\mathrm{SN}$, Hehir M, et al. Multiple transrectal ultrasound-guided prostatic biopsies— true morbidity and patient acceptance. Br I Urol 1993;71:460-3.

13. Djavan B, Waldert M, Zlotta A, et al. Safety and morbidity of first and repeat transrectal ultrasound guided prostate needle biopsies: results of a prospective European prostate cancer detection study. J Urol 2001;166:856-60.

14. Kapoor DA, Klimberg IW, Malek GH, et al. Single-dose oral ciprofloxacin versus placebo for prophylaxis during transrectal prostate biopsy. Urology 1998;52:552-8.

15. Raaijmakers R, Kirkels WJ, Roobol MJ, et al. Complication rates and risk factors of 5802 transrectal ultrasound-guided sextant biopsies of the prostate within a population-based screening program. Urology 2002;60:826-30

16. Ramey JR, Halpern EJ, Gomlla LG. Ultrasonography and Biopsy of the Prostate. In: Wein AJ, Kavoussi LR, Novick AC, Partin AW, Peters CA, eds. Campbell-Walsh Urology. 9th ed. Philadelphia, PA: Saunders; 2002:2883-95. 
17. Sabbagh R, McCormack M, Peloquin F, et al. A prospective randomized trial of 1-day versus 3-day antibiotic prophylaxis for transrectal ultrasound guided prostate biopsy. Can I Urol 2004;11:2216-9.

18. Tal R, Livne PM, Lask DM, et al. Empirical management of urinary tract infections complicating transrectal ultrasound guided prostate biopsy. J Urol 2003;169:1762-5.

19. Shigehara K, Miyagi T, Nakashima T, et al. Acute bacterial prostatitis after transrectal prostate needle biopsy: clinical analysis. I Infect Chemother 2008;14:40-3.

20. Clements R, Aideyan OU, Griffiths GJ, et al. Side effects and patient acceptability of transrectal biopsy of the prostate. Clin Radiol 1993;47:125-6.

21. Nash PA, Bruce JE, Indudhara R, et al. Transrectal ultrasound guided prostatic nerve blockade eases systematic needle biopsy of the prostate. J Urol 1996;155:607-9.

22. Cevik I, Ozveri H, Dillioglugil 0 , et al. Lack of effect of intrarectal lidocaine for pain control during transrectal prostate biopsy: a randomized prospective study. Eur Urol 2002;42:217-20.

23. Trucchi A, De Nunzio C, Mariani S, et al. Local anesthesia reduces pain associated with transrectal prostatic biopsy. A prospective randomized study. Urol Int 2005;74:209-13.

24. Lynn NN, Collins GN, Brown SC, et al. Periprostatic nerve block gives better analgesia for prostatic biopsy. BJU Int 2002;90:424-6.

25. Alavi AS, Soloway MS, Vaidya A, et al. Local anesthesia for ultrasound guided prostate biopsy: a prospective randomized trial comparing 2 methods. J Urol 2001;166:1343-5.

26. Obek C, Onal B, Ozkan B, et al. Is periprostatic local anesthesia for transrectal ultrasound guided prostate biopsy associated with increased infectious or hemorrhagic complications? A prospective randomized trial. J Urol 2002; 168:558-61.

27. Conde Redondo C, Alonso Fernández D, Robles Samaniego A, et al. TRUS-guided biopsy: comparison of two anesthetic methods [in Spanish]. Actas Urol Esp 2006;30:134-8.

28. Bhomi KK, Lim HH, Consigliere DT, et al. Control of pain during transrectal ultrasound-guided prostate biopsy: a prospective study comparing two methods. Urol Int 2007;79:332-5.

29. Haq A, Patel HR, Habib MR, et al. Diclofenac suppository analgesia for transrectal ultrasound guided biopsies of the prostate: a double-blind, randomized controlled trial. J Urol 2004;171:1489-91.

30. Irer B, Gulcu A, Aslan G, et al. Diclofenac suppository administration in conjunction with lidocaine gel during transrectal ultrasoundguided prostate biopsy: prospective, randomized, placebo-controlled study. Urology 2005;66:799-802.

31. Rogatsch H, Moser P, Volgger H, et al. Diagnostic effect of an improved preembedding method of prostate needle biopsy specimens. Hum Pathol 2000;31:1102-7.

32. Kao J, Upton $M$, Zhang $P$, et al. Individual prostate biopsy core embedding facilitates maximal tissue representation. I Urol 2002;168:496-9.

33. Terris MK. Ultrasonography and Biopsy of the Prostate. In: Walsh, P. C., Retik AB, Vaughan ED, Wein AJ, Kavoussi LR, Novick AC, eds. Campbell's Urology. 8th ed. Pennsylvania, PA: WB Saunders; 2002:3054.

34. Taneja SS, Penson DF, Epelbaum A, et al. Does site specific labeling of sextant biopsy cores predict the site of extracapsular extension in radical prostatectomy surgical specimen. J Urol 1999;162:1352-7.

35. Gupta C, Ren IZ, Woino KJ. Individual submission and embedding of prostate biopsies decreases rates of equivocal pathology reports. Urology 2004;63:83-6.

36. Kaye KW. Prostate biopsy using automatic gun. Technique for determination of precise biopsy site. Urology 1989;34:111-2.

37. Frauscher F, Klauser A, Volgger H, et al. Comparison of contrast enhanced color Doppler targeted biopsy with conventional systematic biopsy: impact on prostate cancer detection. J Urol 2002;167:1648-52.

38. Hodge KK, McNeal JE, Terris MK, et al. Random systematic versus directed ultrasound guided transrectal core biopsies of the prostate. J Urol 1989;142:71-4.

39. Eskew LA, Bare RL, McCullough DL. Systematic 5 region prostate biopsy is superior to sextant method for diagnosing carcinoma of the prostate. J Urol 1997:157:199-202.

40. Presti JC Jr, Chang JJ, Bhargava V, et al. The optimal systematic prostate biopsy scheme should include 8 rather than 6 biopsies: results of a prospective clinical trial. J Urol 2000;163:163-6.

41. Norberg M, Egevad L, Holmberg L, et al. The sextant protocol for ultrasound-guided core biopsies of the prostate underestimates the presence of cancer. Urology 1997;50:562-6.

42. Stamey TA. Making the most out of six systematic sextant biopsies. Urology 1995;45:2-12.

43. Fink KG, Hutarew $G$, Pytel A, et al. One 10-core prostate biopsy is superior to two sets of sextant prostate biopsies. BJU Int 2003;92:385-8.

44. Durkan GC, Sheikh N, Johnson P, et al. Improving prostate cancer detection with an extended-core transrectal ultrasonographyguided prostate biopsy protocol. BJU Int 2002;89:33-9.

45. Brossner $C$, Bayer $G$, Madersbacher $S$, et al. Twelve prostate biopsies detect significant cancer volumes (>0.5 mL). BJU Int 2000;85:705-7.

46. Babaian RJ, Toi A, Kamoi K, et al. A comparative analysis of sextant and an extended 11-core multisite directed biopsy strategy. J Urol 2000;163:152-7.

47. Chang JJ, Shinohara K, Bhargava V, et al. Prospective evaluation of lateral biopsies of the peripheral zone for prostate cancer detection. J Urol 1998;160:2111-4.
48. Eichler K, Hempel S, Wilby J, et al. Diagnostic value of systematic biopsy methods in the investigation of prostate cancer: a systematic review. J Urol 2006;175:1605-12.

49. Pepe P, Aragona F. Saturation prostate needle biopsy and prostate cancer detection at initial and repeat evaluation. Urology 2007;70:1131-5

50. Toi A, Neill MG, Lockwood GA, et al. The continuing importance of transrectal ultrasound identification of prostatic lesions. J Urol 2007; 177:516-20.

51. Applewhite JC, Matlaga BR, McCullough DL, et al. Transrectal ultrasound and biopsy in the early diagnosis of prostate cancer. Cancer Control 2001;8:141-50.

52. Uzzo RG, Wei JT, Waldbaum RS, et al. The influence of prostate size on cancer detection. Urology 1995;46:831-6.

53. Levine MA, Ittman M, Melamed J, et al. Two consecutive sets of transrectal ultrasound guided sextant biopsies of the prostate for the detection of prostate cancer. J Urol 1998;159:471-5.

54. Karakiewicz Pl, Bazinet M, Aprikian AG, et al. Outcome of sextant biopsy according to gland volume. Urology 1997; 49:55-9

55. Applewhite IC, Metwalli AR, McCullough DL. Results of the five-region prostate biopsy method: the effect of gland size and number of cores on yield. Proceedings of the 64th Annual Meeting of the Southeastern Section of the American Urological Association; March 30 - April 2, 2000; Orlando, FL.

56. Remzi $M$, Fong $Y K$, Dobrovits $M$, et al. The Vienna nomogram: validation of a novel biopsy strategy defining the optimal number of cores based on patient age and total prostate volume. J Urol 2005;174:1256-60.

57. Epstein Jl, Walsh PC, Sauvageot J, et al. Use of repeat sextant and transition zone biopsies for assessing extent of prostate cancer. J Urol 1997; 158:1886-90.

58. Terris MK, Pham TQ, Issa MM, et al. Routine transition zone and seminal vesicle biopsies in all patients undergoing transrectal ultrasound guided prostate biopsies are not indicated. J Urol 1997;157:204-6.

59. Fleshner NE, Fair WR. Indications for transition zone biopsy in the detection of prostatic carcinoma. J Urol 1997; 157:556-8.

60. Bazinet $M$, Karakiewicz Pl, Aprikian AG, et al. Value of systematic transition zone biopsies in the early detection of prostate cancer. J Urol 1996; 155:605-6.

61. Hong YM, Lai FC, Chon $\mathrm{CH}$, et al. Impact of prior biopsy scheme on pathologic features of cancers detected on repeat biopsies. Urol Oncol 2004;22:7-10.

62. Haussler 0, Epstein Jl, Amin MB, et al. Cell proliferation, apoptosis, oncogene, and tumor suppressor gene status in adenosis with comparison to benign prostatic hyperplasia, prostatic intraepithelial neoplasia, and cancer. Hum Pathol 1999;30:1077-86.

63. O'dowd GJ, Miller MC, Orozco $R$, et al. Analysis of repeated biopsy results within 1 year after a noncancer diagnosis. Urology 2000;55:553-9.

64. Shepherd D, Keetch DW, Humphrey PA, et al. Repeat biopsy strategy in men with isolated prostatic intraepithelial neoplasia on prostate needle biopsy. J Urol 1996;156:460-2.

65. Allen EA, Kahane H, Epstein Jl. Repeat biopsy strategies for men with atypical diagnoses on initia prostate needle biopsy. Urology 1998;52:803-7.

66. Park S, Shinohara K, Grossfeld GD, et al. Prostate cancer detection in men with prior high grade prostatic intraepithelial neoplasia or atypical prostate biopsy. J Urol 2001;165:1409-14.

67. Kronz JD, Shaikh AA, Epstein JI. High-grade prostatic intraepithelial neoplasia with adjacent small atypical glands on prostate biopsy. Hum Pathol 2001;32:389-95.

68. Lefkowitz GK, Sidhu GS, Torre P, et al. Is repeat prostate biopsy for high-grade prostatic intraepithelial neoplasia necessary after routine 12-core sampling? Urology 2001;58:999-1003.

69. Mian BM, Naya Y, Okihara K, et al. Predictors of cancer in repeat extended multisite prostate biopsy in men with previous negative extended multisite biopsy. Urology 2002;60:836-40.

70. Moore CK, Karikehalli S, Nazeer T, et al. Prognostic significance of high grade prostatic intraepithelial neoplasia and atypical small acinar proliferation in the contemporary era. J Urol 2005:173:70-2.

71. Bostwick DG, Srigley J, Grignon D, et al. Atypical adenomatous hyperplasia of the prostate: morphologic criteria for its distinction from well-differentiated carcinoma. Hum Pathol 1993;24:819-32.

72. Amin $M$, Jeyaganth S, Fahmy N, et al. Subsequent prostate cancer detection in patients with prostatic intraepithelial noeplasia or atypical small acinar proliferation. Can Urol Assoc J 2007; 1:245-9.

73. Iczkowski KA, MacLennan GT, Bostwick DG. Atypical small acinar proliferation suspicious for malignancy in prostate needle biopsies: clinical significance in 33 cases. Am I Surg Pathol 1997;21:1489-95.

74. Stewart CS, Leibovich BC, Weaver AL, et al. Prostate cancer diagnosis using a saturation needle biopsy technique after previous negative sextant biopsies. J Urol 2001;166:86-91.

75. Borboroglu PG, Comer SW, Riffenburgh RH, et al. Extensive repeat transrectal ultrasound guided prostate biopsy in patients with previous benign sextant biopsies. J Urol 2000;163:158-62.

76. Igel TC, Knight MK, Young PR, et al. Systematic transperineal ultrasound guided template biopsy of the prostate in patients at high risk. J Urol 2001;165:1575-9.

Correspondence: Dr. Assaad El-Hakim, Assistant Professor of Urology, McGill University, 175 Ch. Stillview, Suite 200, Pointe-Claire, QC H9R 4S3; fax: 514-695-0431; assaad.elhakim@mcgill.ca 